\title{
Switch-to-Semaglutide Study (STS-Study): a Retrospective Cohort Study
}

\author{
Matthias Hepprich (D) - Daniela Zillig - Manuel A. Florian-Reynoso · \\ Marc Y. Donath · Gottfried Rudofsky
}

Received: December 20, 2020 / Accepted: January 29, 2021 / Published online: February 22, 2021

(C) The Author(s) 2021

\section{ABSTRACT}

Introduction: Despite expert consensus guidelines, data is scarce on how to switch patients with type 2 diabetes when treatment with glucagon-like peptide 1 (GLP-1) receptor agonists is not effective and whether a switch to semaglutide brings any benefit on glucose and weight control for patients with type 2 diabetes.

Methods: Retrospective cohort analysis of patients with type 2 diabetes who were switched from any GLP-1 agonist in a stable dose to subcutaneously administered semaglutide. Primary endpoint was change of glycated haemoglobin (HbA1c) at 6 months. Secondary

Supplementary Information The online version contains supplementary material available at https:// doi.org/10.1007/s13300-021-01016-y.

M. Hepprich $(\bowtie) \cdot$ D. Zillig · G. Rudofsky Clinic of Endocrinology and Metabolic Disorders, Cantonal Hospital Olten, Baslerstrasse 150, Olten, Switzerland

e-mail: matthias.hepprich@usb.ch

M. A. Florian-Reynoso

Medical Faculty, University of Basel,

Klingelbergstrasse 61, Basel, Switzerland

M. Hepprich · M. Y. Donath

Clinic of Endocrinology, Diabetes and Metabolism, University Hospital Basel, Petersgraben 4, Basel,

Switzerland endpoints were weight, body mass index (BMI), heart rate, blood pressure and adverse events.

Results: In total, 77 patients (median age 65 years) with long-standing type 2 diabetes (median 15 years, median HbA1c 8.4\%/ $68 \mathrm{mmol} / \mathrm{l}$, median BMI $33 \mathrm{~kg} / \mathrm{m}^{2}$ ) were included. HbA1c was significantly lower 6 months after switching to semaglutide $(7.3 \% ; 56 \mathrm{mmol} /$ 1). Median body weight was significantly lower at 3 months $(94 \mathrm{~kg})$ and 6 months $(93 \mathrm{~kg})$ compared to baseline $(98 \mathrm{~kg})$. An equipotential dose switch of semaglutide was used in 61 patients (79\%) and a stepwise initiation approach was used in 16 patients (21\%). Both treatment regimens improved glucose control and weight. Side effects occurred in 28 patients (36\%).

Conclusion: Switching to semaglutide from established GLP-1 analogue therapy improved HbA1c and body weight. Both equipotential and stepwise dosing initiation appear to be effective and well tolerated.

Keywords: Antidiabetic drug; GLP-1 analogue; Observational study; Semaglutide; Type 2 diabetes 


\section{Key Summary Points}

\section{Why carry out this study?}

GLP-1 agonists are effective in controlling type 2 diabetes but tend to lose efficacy over time.

Subcutaneously administered semaglutide is more efficacious in head-to-head studies compared with other GLP-1 agonists but data on switching GLP-1 agonist therapy to semaglutide is lacking.

We therefore investigated real-world efficacy of a treatment switch from GLP-1 agonists to semaglutide on glucose control and weight in patients with type 2 diabetes.

\section{What was learned from the study?}

Switching patients with pre-treated type 2 diabetes from GLP-1 analogue to subcutaneously administered semaglutide significantly reduces $\mathrm{HbA} 1 \mathrm{c}$ and body weight over 6 months compared to baseline.

Equivalent dosing of semaglutide is effective, well tolerated and may be preferred over a stepwise dosing regimen when switching from other GLP-1 agonists.

\section{DIGITAL FEATURES}

This article is published with digital features, including a summary slide, to facilitate understanding of the article. To view digital features for this article go to https://doi.org/10.6084/ m9.figshare.13655921.

\section{INTRODUCTION}

Despite recent expert recommendations robust clinical data on treatment switches of glucagon- like peptide 1 (GLP-1) receptor agonists is scarce $[1,2]$. GLP-1 agonists are an important cornerstone for the treatment of type 2 diabetes [3] and have been proven to be highly efficacious not only for glucose control but also for weight loss $[4,5]$. They have been proven to be safe and to be beneficial for the reduction of cardiovascular events [6, 7]. However, from clinical experience GLP-1 analogues show decreasing effects on glycaemic and weight control over time and therefore require treatment adaptations.

Since September 2018, a new candidate of once weekly subcutaneously administered GLP1 analogues, semaglutide, amended the medical options for treating type 2 diabetes in Switzerland. In head-to-head studies with other GLP-1 analogues, semaglutide was more efficacious with respect to glucose control and weight reduction in patients with type 2 diabetes [8-11]. However, to date, there is-apart from a letter to the editor by Goncalves and Bell [12]no data for efficacy and safety when patients are treated with any type of GLP-1 agonist whether a switch to semaglutide is effective. Furthermore, it is unknown which starting dose might be suitable in such patients. For semaglutidenaive patients, a stepwise dosing approach starting with $0.25 \mathrm{mg}$ once weekly is recommended. Whether this is necessary for patients already treated with a GLP-1 agonist is unclear. Theoretical exposure-response modelling by Overgaard et al. based on semaglutide phase III study data suggests an additional benefit for glucose control as well as for further weight loss [13]. Additionally, the model provides an estimate of corresponding GLP-1 levels for liraglutide, dulaglutide and exenatide in comparison to semaglutide dosing for a treatment switch which would allow an equipotential dosing switch. However, whether these theoretical estimations hold true in clinical practice and whether gastrointestinal tolerability or other side effects are comparable when patients are switched from a pre-existing GLP-1 analogue therapy to semaglutide are unclear $[1,2]$.

Therefore, the aim of our retrospective study was to systematically investigate the efficacy of glucose control and weight change over 6 months of a switch to semaglutide from any 
other GLP-1 analogue therapy in a real-world clinical practice setting.

\section{METHODS}

This retrospective cohort study was performed at two Swiss endocrine outpatient clinics (University Hospital Basel and Cantonal Hospital Olten). Patients were included in the study if they had a diagnosis of type 2 diabetes according to the current American Diabetes Association (ADA) guidelines, an age of 18 years or older, unchanged diabetes medication with a GLP-1 analogue (liraglutide, dulaglutide, lixisenatide, exenatide) for at least 3 months or longer, and a switch to semaglutide was performed and follow-up data for at least 6 months after switching to semaglutide were available. Patients were excluded if they had any other type of diabetes or when a significant glucosealtering medication, such as glucocorticosteroids or diabetes medication, was initiated simultaneously to semaglutide.

The primary endpoint was the change in glycated haemoglobin A1c (HbA1c) at 6 months after switch to semaglutide compared to baseline. Secondary endpoints were $\mathrm{HbA1c}$ at 3 months as well as weight change, change in body mass index (BMI), heart rate and blood pressure; type and frequency of gastrointestinal symptoms; and change in antidiabetic medication at 3 and 6 months.

At the University Hospital Basel, an electronic database search was performed to retrospectively identify patients for whom a switch of any commonly used GLP-1 analogue to semaglutide was performed. All patient data of the endocrine outpatient clinic from January 1, 2018, until December 31, 2019, were included in the search with hits for the trade names of "semaglutide" and "liraglutide" or "dulaglutide" or "exenatide". Trade names would be regularly used in the patient record forms instead of compound names. Only data from subjects with a signed general consent form were included in the data search.

At the endocrine outpatient clinic at the Cantonal Hospital Olten, all patients who were switched to semaglutide were asked retrospectively to participate in the study either directly during routine outpatient clinic consultations or via phone call. All participants were required to sign an informed consent form for study participation.

In case, follow-up data was not available inhouse, patients and their consecutive treating physicians were contacted to obtain all missing data.

As a comparator, HbA1c values from 3 to 6 months before treatment switch to semaglutide of the same patients were used.

To minimize selection bias, the participant study population was clearly defined and HbA1c values before treatment switch of the same cohort were used.

\section{Statistical Analysis}

On the basis of the data of Overgaard et al. [13] and an estimated effect size of a $0.4 \%$ difference in the HbA1c change, a sample size of 75 patients was calculated to be sufficient given an alpha of 0.05 and a power of 0.95 . A non-normal distribution was assumed and therefore Wilcoxon matched-pairs signed rank test was used for sample size calculation. The sample size was calculated with $G^{*}$ Power (University of Düsseldorf, version 3.1.9.4).

Descriptive statistics with mean and 95\% confidence interval as well as median and interquartile range were used for continuous variables. Data were assessed for normal distribution using mean, median, skewness, kurtosis and D'Agostino and Pearson test. For parametric data of two groups Student's $t$ test was used. For nonparametric paired data of two groups, Wilcoxon matched-pairs signed rank test was used. An $\alpha$ of less than 0.05 was regarded as statistically significant. Missing data were not imputed and regarded as missing at random. Data were analysed using Graph Pad Prism Version 8.2.1 for Mac OS X.

\section{Ethics and Transparency}

The study was conducted in compliance with the current version of the Declaration of Helsinki, the ICH-GCP and national legal and 


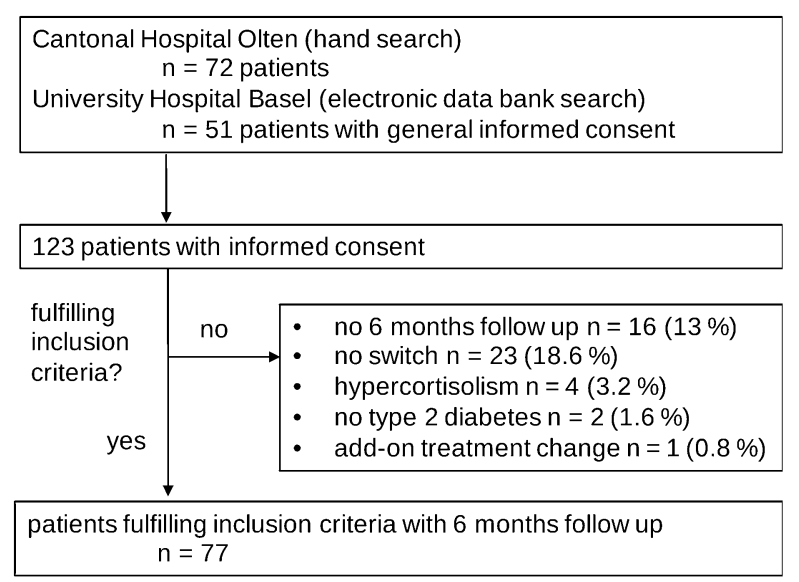

Fig. 1 Study flow chart

regulatory requirements. The study was approved by the local ethics committee (EKNZ 2019-01770).

The dataset will be provided online after publication in the Open Science Framework.

\section{RESULTS}

Data were collected between January 1, 2020, and June 30, 2020. The data search yielded 139 potential patients at both endocrine outpatient clinics, of whom 123 gave written informed consent. Of these, 77 patients fulfilled all inclusion criteria for further data analysis, with $71(92 \%)$ patients at 3 months and $77(100 \%)$ patients at 6 months (Fig. 1). The most common reasons for not fulfilling the inclusion criteria were that effectively no switch to semaglutide had been done in 23 patients $(18.6 \%)$ and that no follow-up data at 3 and 6 months were available (16 patients, 13\%).

Baseline characteristics are displayed in Table 1. In brief, patients had a median age of 65 years, a median body weight of $96 \mathrm{~kg}$ (median BMI of $33 \mathrm{~kg} / \mathrm{m}^{2}$ ) and 27 patients (35\%) were female. The median duration of diabetes was 15 years (range $2-37$ years) with a median HbA1c of $8.4 \%$ (68 mmol/l). In 57 patients (74\%) diabetes-related complications were known. Patients were pre-treated for a median of 25 months with GLP-1 analogues, some for up to 6 years. Most of the patients were treated with liraglutide ( $n=61,79 \%$ ). Dulaglutide was used in 11 patients (14\%), exenatide in three patients (3.8\%) and two patients received orally administered semaglutide $(n=2,2.6 \%$, PIONEER 7 study participants, NCT02849080) before switching to semaglutide. The majority of patients $(n=61,79 \%)$ received additional treatment with metformin, 16 patients $(20 \%)$ with sulfonylureas, and 8 patients $(10 \%)$ with sodium-glucose cotransporter 2 (SGLT2) inhibitors. In 56 patients (72\%), basal insulin was used (median daily basal insulin dose of $36 \mathrm{IU}$ ), and 12 patients $(15 \%)$ required bolus insulin therapy (median daily prandial insulin dose of $37 \mathrm{IU})$. Statins and treatment with angiotensinconverting enzyme (ACE) inhibitors or angiotensin II receptor blockers (ARBs) were used in 59 patients (76\%).

First, the effect on glucose control was analysed. Compared to baseline median $\mathrm{HbA1c}$ was significantly lower $(7.3 \%$, interquartile range [IQR] 6.6-8.1; $56 \mathrm{mmol} / \mathrm{mol}$, IQR 48-65) 6 months after switching to semaglutide compared to baseline (8.4\%, IQR 7.7-9.3, 68 mmol/mol, IQR 60-78; Fig. 2a). In comparison, HbA1c did not significantly change within 3-6 months from pre-baseline $(8.3 \%$, IQR $7.4-9.2 \%, 67 \mathrm{mmol} / \mathrm{mol}$, IQR $57-77, n=68$ ) to baseline.

However, median HbA1c was already significantly lower at 3 months (7.7\%, IQR 6.9-8.3\%; $60 \mathrm{mmol} / \mathrm{mol}$, IQR 51-67) compared to baseline and absolute median body weight was significantly lower at 3 months (94 kg, IQR 83-111 kg) 
Table 1 Baseline characteristics of all 77 patients

\begin{tabular}{|c|c|c|c|c|}
\hline Characteristic & $\begin{array}{l}\text { Median } \\
\text { (IQR; range) }\end{array}$ & $\begin{array}{l}\text { Mean } \\
(95 \% \mathrm{CI})\end{array}$ & $n(\%)$ & Missing values \\
\hline Age & $65(56-70 ; 32-85)$ & $61(61-66)$ & & 0 \\
\hline \multicolumn{5}{|l|}{ Sex } \\
\hline Female & & & $27(35 \%)$ & 0 \\
\hline Male & & & $50(64 \%)$ & 0 \\
\hline Body weight $(\mathrm{kg})$ & $96(86-113 ; 67-172)$ & $101(96-106)$ & & 1 \\
\hline Body mass index $\left(\mathrm{kg} / \mathrm{m}^{2}\right)$ & $33(30-39 ; 25-59)$ & $35(34-37)$ & & 1 \\
\hline Systolic blood pressure $(\mathrm{mmHg})$ & $136(124-146 ; 102-198)$ & $137(133-142)$ & & 10 \\
\hline Diastolic blood pressure $(\mathrm{mmHg})$ & $80(76-88 ; 55-110)$ & $81(79-84)$ & & 10 \\
\hline Duration of type 2 diabetes (years) & $15(8.3-21 ; 2-37)$ & $15(13-17)$ & & 1 \\
\hline Diabetes complications & & & $57(74 \%)$ & 0 \\
\hline Macrovascular & & & $17(22 \%)$ & \\
\hline Coronary heart disease & & & $14(18 \%)$ & \\
\hline Cerebrovascular disease & & & $3(3.9 \%)$ & \\
\hline Microvascular & & & $55(71 \%)$ & \\
\hline Retinopathy & & & $15(19 \%)$ & \\
\hline Neuropathy & & & $35(45 \%)$ & \\
\hline Nephropathy & & & $35(45 \%)$ & \\
\hline $\begin{array}{l}\text { Duration of preceding GLP-1 } \\
\text { analogue therapy (months) }\end{array}$ & $25(18-41 ; 3-72)$ & $28(25-32)$ & & 5 \\
\hline \multicolumn{5}{|l|}{ Glycated haemoglobin Alc (HbAlc) } \\
\hline$\%$ & $8.4(7.7-9.3 ; 5.0-12)$ & $8.3(8.0-8.6)$ & & 0 \\
\hline $\mathrm{mmol} / \mathrm{mol}$ & $68(60-78 ; 31-107)$ & $67(63-70)$ & & \\
\hline Preceding GLP-1 analogue & & & & 0 \\
\hline Liraglutide & & & $61(79.2 \%)$ & \\
\hline Dulaglutide & & & $11(14.0 \%)$ & \\
\hline Exenatide & & & $3(3.8 \%)$ & \\
\hline Orally administered semaglutide & & & $2(2.6 \%)$ & \\
\hline
\end{tabular}


Table 1 continued

\begin{tabular}{|c|c|c|c|c|}
\hline Characteristic & $\begin{array}{l}\text { Median } \\
\text { (IQR; range) }\end{array}$ & $\begin{array}{l}\text { Mean } \\
(95 \% \mathrm{CI})\end{array}$ & $n(\%)$ & Missing values \\
\hline $\begin{array}{l}\text { Concomitant oral diabetes } \\
\text { medication metformin }\end{array}$ & & & $61(79 \%)$ & \\
\hline Metformin dose (g) & $2.0(1.0-2.0 ; 0.5-3.0)$ & $1.7(1.6-1.9)$ & & \\
\hline Pioglitazone & & & $4(5.2 \%)$ & \\
\hline Sulfonyl urea & & & $16(20.7 \%)$ & \\
\hline SGLT2 inhibitor & & & $8(10.3 \%)$ & \\
\hline \multicolumn{5}{|l|}{ Concomitant insulin therapy } \\
\hline Basal insulin & & & $56(72.7 \%)$ & \\
\hline Daily basal insulin dose (IU) & $36(30-50 ; 12-145)$ & $44(37-51)$ & & \\
\hline Prandial insulin & & & $12(15.5 \%)$ & \\
\hline Daily prandial insulin dose (IU) & 37 (29-44; 12-85) & $37(27-48)$ & & \\
\hline \multicolumn{5}{|l|}{ Concomitant therapy } \\
\hline Aspirin & & & $37(48.0 \%)$ & \\
\hline Statin & & & $62(80.5 \%)$ & \\
\hline $\mathrm{ACE} / \mathrm{ARB}$ inhibitor & & & $59(76.6 \%)$ & \\
\hline
\end{tabular}

Given are median values with interquartile range (IQR), range as well as mean with $95 \%$ confidence interval (CI), the number with percentage and number of missing values

and 6 months (93 kg, IQR $81-110 \mathrm{~kg}$ ) after switching to semaglutide compared to baseline (Fig. 2b) which resulted also in a significant reduction in BMI at 3 and 6 months compared to baseline (Fig. 2c for details).

However, there was no significant change in systolic or diastolic blood pressure and heart rate 3 months after switching to semaglutide (data not shown).

The median basal daily insulin requirement was not significantly different at 3 months (insulin dose $40 \mathrm{IU} /$ day; IQR 30-60 IU/day) and 6 months (insulin dose 39 IU/day; IQR 27-$60 \mathrm{IU} /$ day) after switching to semaglutide. Prandial insulin was required in eight patients at 3 months and in five patients at 6 months after switching to semaglutide compared to 12 patients at baseline. The median prandial insulin dose did not significantly change at 3 months (insulin dose 30 IU/day; IQR 28-44) or 6 months (insulin dose $29 \mathrm{IU} /$ day; IQR 17-59) after treatment switch (see Supplementary Fig. 1 for details).

In eight patients, changes in the remaining diabetes medication were observed after the treatment switch. In two patients, SGLT2 inhibitors were stopped because of urinary tract infections. In one patient, gliclazide was discontinued with initiation of semaglutide. In another patient, pioglitazone was discontinued shortly after the switch to semaglutide. In two patients, metformin, in one patient pioglitazone and in another patient gliclazide were added to the current diabetes medication. When all patients with changes of their diabetes medication and all patients who received additional SGLT2 inhibitor treatment were excluded from the analysis, HbA1c and weight were still significantly lower at 6 months after treatment switch (mean HbA1c 7.5\%, $69 \mathrm{mmol} / \mathrm{mol}$, body 
A

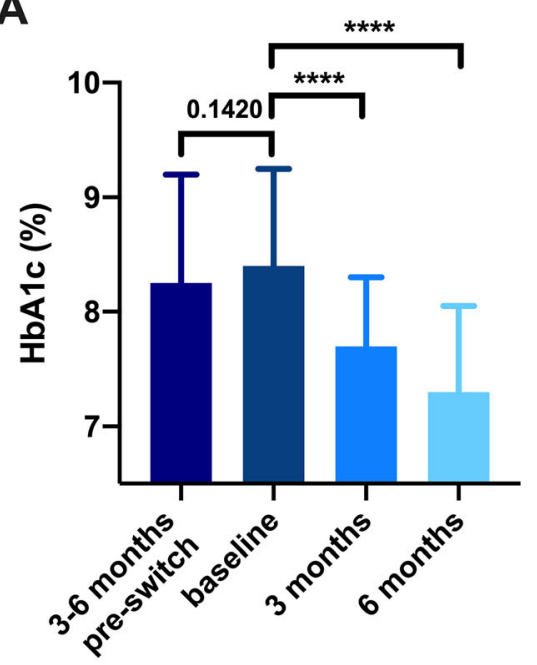

B

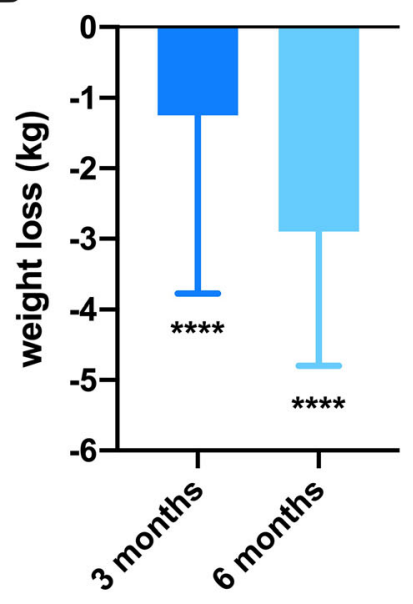

C

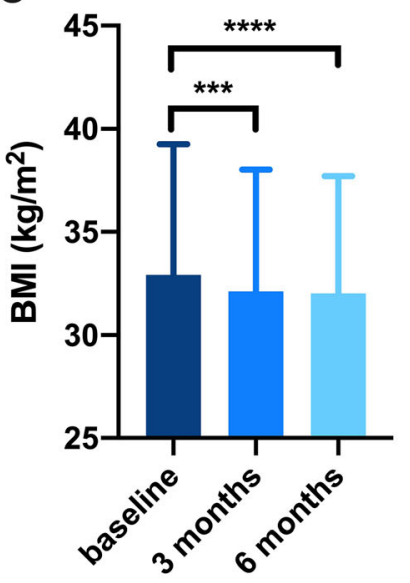

Fig. $2 \mathrm{HbAlc}$ and weight change. a Absolute mean HbAlc was $8.5 \%$ (95\% CI 8.2-8.7\%) at baseline and 7.7\% (95\% CI 7.4-8.0\%) and 7.5\% (95\% CI 7.2-7.7\%) at 3 and 6 months, respectively, in comparison to pre-baseline (8.3\%, 95\% CI 8.0-8.6\%). b Mean weight loss was $1.6 \mathrm{~kg}(95 \%$ CI $2.4-0.91 \mathrm{~kg})$ and $3.0 \mathrm{~kg}(95 \% \mathrm{CI} 4-2 \mathrm{~kg})$ at 3 and 6 months, respectively after treatment with semaglutide compared to baseline. c BMI was significantly lower at 3 months (mean $35 \mathrm{~kg} / \mathrm{m}^{2} 95 \%$ CI $33-36 \mathrm{~kg} / \mathrm{m}^{2}$ ) and 6 months $\left(34 \mathrm{~kg} / \mathrm{m}^{2} 95 \%\right.$ CI $\left.33-36 \mathrm{~kg} / \mathrm{m}^{2}\right)$ after switching to semaglutide compared to baseline (mean $35 \mathrm{~kg} / \mathrm{m}^{2}, 95 \%$ CI $\left.34-37 \mathrm{~kg} / \mathrm{m}^{2}\right)$. Graphically represented are median values and interquartile range. $\mathrm{CI}$ confidence interval, ${ }^{* * *} p<0.005,{ }^{* * * *} p<0.0001$ weight $97 \mathrm{~kg}$ ) compared to baseline (HbA1c $8.5 \%, 58 \mathrm{mmol} / \mathrm{mol}$, body weight $100 \mathrm{~kg}$; see Supplementary Table 2).

Furthermore, we performed subgroup analyses for different treatment regimes. In the Basel cohort, all patients except for one were initiated according to the label starting with semaglutide $0.25 \mathrm{mg}$ qw (i.e. standard dosing group), whereas in the Olten cohort all patients were switched to semaglutide according to exposure-response modelling by Overgaard et al. [13] (equipotential dosing group). All patients in both groups received from 3 months onwards the maximum recommended dose of semaglutide of $1 \mathrm{mg}$ per week unless semaglutide was discontinued $(n=3)$. The two groups did not significantly differ regarding age, duration of diabetes or weight. However, patients in the equipotential dosing group had a lower baseline HbA1c than the standard dosing group (HbA1c $8.0 \%$ vs. $8.9 \% ; 63 \mathrm{mmol} / \mathrm{mol}$ vs. $73 \mathrm{mmol} / \mathrm{mol}$, $p=0.02)$.

In the standard dosing group, HbA1c was significantly lower at 3 months (median HbA1c 8.1\%, IQR 6.9-8.6; $65 \mathrm{mmol} / \mathrm{mol}$, IQR 51-70) compared to baseline and showed a statistically nonsignificant albeit clinically meaningful reduction of median $\mathrm{HbA1c}$ at 6 months (HbA1c 7.5\%, IQR 7.3-9.3; $58 \mathrm{mmol} / \mathrm{l}$, IQR 56-78) (Fig. 3). In the equipotential dosing group, HbA1c was significantly lower at 3 months (HbA1c 7.6\%, IQR 6.8-8.1; $59 \mathrm{mmol} / \mathrm{mol}$, IQR $50-65)$ and at 6 months (HbA1c 7.3\%, IQR 6.5-8.0; $56 \mathrm{mmol} / \mathrm{mol}, \mathrm{IQR}$ 47-63) after switching to semaglutide.

In the standard dosing group, the median weight did not significantly change at 3 months (weight $92 \mathrm{~kg}$; IQR $79-107 \mathrm{~kg}, p=0.65$ ) but showed a trend toward lower body weight at 6 months (weight $90 \mathrm{~kg}$; IQR $80-112 \mathrm{~kg}$, $p=0.06$ ). In the equipotential dosing group, the weight was significantly lower at 3 and 6 months (Fig. 3).

In addition, $\mathrm{HbA} 1 \mathrm{c}$ and weight were significantly lower 6 months after switching to semaglutide from liraglutide or dulaglutide compared to baseline (Supplementary Table 2). HbA1c and weight were not significantly lower at 6 months in patients that were switched from 
A

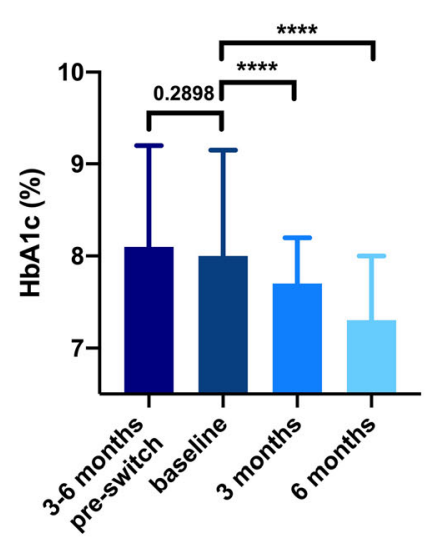

B

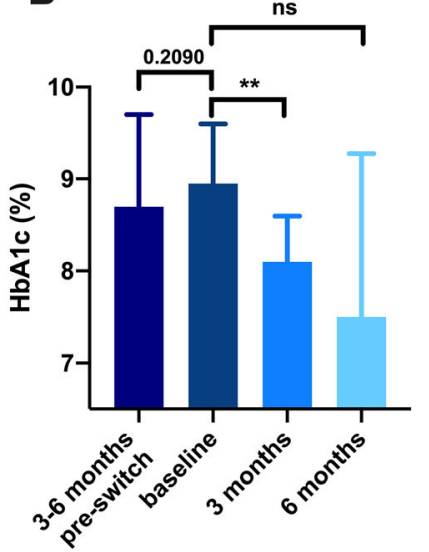

C

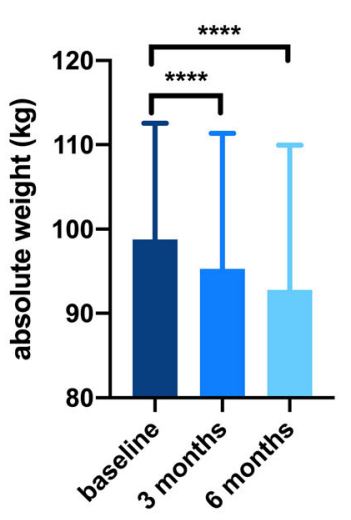

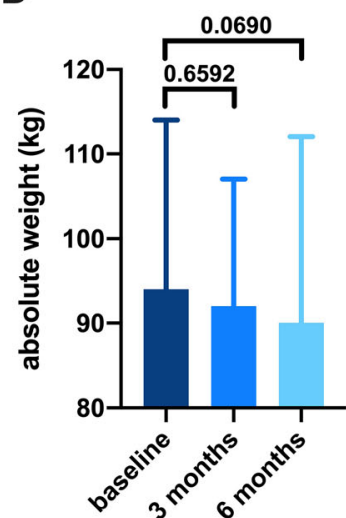

Fig. 3 Comparison of $\mathrm{HbAlc}(\mathbf{a}, \mathbf{b})$ and weight change (c, d) in different switching regimens from GLP-1 analogue treatment to semaglutide. a, c Depict equipotential dosing and $\mathbf{b}, \mathbf{d}$ depict standard dosing according to the label. a Absolute mean HbAlc was $8.2 \%$ (95\% CI 7.8-8.5\%), 8.3\% (95\% CI 8.0-9.7\%), 7.6\% (95\% CI $7.4-7.9 \%)$ and $7.3 \%$ (95\% CI 7.0-7.5\%) 3-6 months prebaseline, at baseline and 3 and 6 months after equipotential treatment switch to semaglutide respectively. b Absolute mean $\mathrm{HbAlc}$ was $8.8 \%$ (95\% CI 8.0-9.6\%), 9.0\% (95\% CI $8.4-9.5 \%), 8.0 \%$ (95\% CI 7.3-8.7\%) and $8.2 \%$

exenatide to semaglutide (Supplementary Table 2).

\section{Adverse Events}

Adverse events occurred in 28 patients (36\%) and are outlined in Supplementary Table 1 . The most common adverse events were nausea $(n=5 ; 6.4 \%)$, vomiting $(n=4 ; 5.2 \%)$ and diarrhoea $(n=5,6.4 \%)$. Two patients $(2.4 \%)$ had to be hospitalized because of gastrointestinal symptoms (gastritis and biliary cholangitis). Semaglutide was discontinued because of side effects in three patients (3.7\%; the earliest after 2 weeks and the latest at 6 months after the treatment switch). All of these patients were treated according to the equipotential dosing regimen. Light to moderate hypoglycaemic episodes occurred in three patients (3.7\%), all of whom were additionally treated with insulin. In two of these patients, hypoglycaemia occurred when they had diarrhoea. No patient died
(95\% CI 7.3-9.1) 3-6 months pre-baseline, at baseline and 3 and 6 months after standard dosing treatment switch to semaglutide respectively. c Absolute mean weight in $\mathrm{kg}$ was 102 (95\% CI 96-108), 101 (95\% CI 94-107) and 99 $(95 \%$ CI $92-106 \mathrm{~kg})$ at baseline, 3 and 6 months after equipotential treatment switch to semaglutide respectively. d Absolute mean weight in $\mathrm{kg}$ was 96 (95\% CI 87-106), 94 (95\% CI 94-104), 93 (95\% CI 93-104). Graphically represented are median values and interquartile range. CI confidence interval, ns non-significant, ${ }^{* * *} p<0.005$, ${ }^{* * * *} p<0.0001$

during the observation period. However, one patient with long-standing diabetes had a myocardial infarction.

\section{DISCUSSION}

In this retrospective cohort study, we show that switching patients with type 2 diabetes from an ongoing GLP-1 analogue therapy to semaglutide (1 $\mathrm{mg}$ weekly) is highly efficacious in improving HbA1c and lowering body weight within 6 months.

Most of the patients in our cohort had longstanding diabetes of more than 10 years, and more than $50 \%$ had been treated for 2 years or more with GLP-1 analogues. However, the diabetes treatment at baseline was not sufficiently controlled, as reflected in the median baseline HbA1c level of $8.4 \%(68 \mathrm{mmol} / \mathrm{mol})$, despite intensified therapy including insulin in $72 \%$ of the patients. Treatment with semaglutide improved the HbA1c level at 3 and 6 months, 
with median reductions of $0.7 \%$ and $0.9 \%$, respectively. As a comparator, we used HbA1c values 3-6 months pre-baseline from the same cohort. These HbA1c values were similar to baseline, indicating that the observed effect is primarily due to the treatment change with semaglutide.

In our cohort, treatment with semaglutide reduced median body weight by $1.3 \mathrm{~kg}$ and $2.9 \mathrm{~kg}$ over 3 and 6 months, respectively. These observations are in line with the theoretical estimations of Overgaard et al. [13], who suggested $\mathrm{HbA1c}$ reductions from $0.3 \%$ to $0.8 \%$ and a body weight loss of $2-4 \%$ within a year [13]. Interestingly, the baseline characteristics of Overgaard et al.'s patients were very similar to the studied population in this analysis (mean baseline HbA1c $8.3 \%$ [67 mmol/mol], body weight of $95 \mathrm{~kg}, 50 \%$ male) [13].

More importantly, both dosing regimens (equipotential and standard) are effective in reducing HbA1c as well as weight even though the small sample size of the standard group may account for the, in part, non-statistically significant but clinically meaningful results for HbA1c and weight at 3 and 6 months.

To date, only limited data exist for switching GLP-1 analogue therapy to semaglutide-containing treatment even though practical and expert consensus recommendations based on empirical and parallel or case-control data were recently published $[1,2]$. To the best of our knowledge, only a letter to the editor analysing a switch from liraglutide to semaglutide has been published so far [12]. These patients had long-standing diabetes and were treated for more than 4 years with liraglutide. Their diabetes control was improved with a reduction of $\mathrm{HbA} 1 \mathrm{c}$ from $7.4 \%$ to $6.6 \%$ [57-48 $\mathrm{mmol} / \mathrm{mol}$, which is similar to the results reported here. However, the analysis included only a small sample size of 40 patients with no detailed data concerning the mode of switch to semaglutide. Furthermore, it only included patients with liraglutide as pretreatment; therefore, the conclusions are rather limited to some extent [12].

Possible explanations for the observed treatment effects after switching from GLP-1 analogues to semaglutide on HbA1c and weight reduction might be multiple. The reasons may in part be explained by a more favourable way of administration (once weekly vs. daily) which may increase patient adherence. Additionally, different pharmacodynamic effects given the substantially varying biochemical properties and thereby a different effect on appetite, satiety, gastric emptying and insulin compared to the respective GLP-1 analogues are likely causes for the results observed in this study $[11,14,15]$.

With respect to side effects, the treatment switch to semaglutide was generally well tolerated in our study, and the number of documented gastrointestinal adverse events was low ( $n=11,14.2 \%)$. This may be in part explained by the retrospective nature and chart analysis of this study. Further, patients who already received GLP-1 analogue treatment may have better tolerated a switch to semaglutide, and furthermore, GLP-1-intolerant patients had already discontinued GLP-1 therapy previously because they could not tolerate their previous GLP-1 analogue-both reasons leading to less frequent side effects compared to those in other semaglutide studies [16, 17]. Nevertheless, treatment with semaglutide had to be discontinued because of side effects in three patients $(3.7 \%)$, all of whom were in the equipotential dosing group, possibly indicating a lower tolerability of this approach. However, the higher discontinuation rate may also be in part explained by the fourfold-larger sample size that was treated according to this regimen.

Our study has limitations. First, it is a retrospective analysis with data from two endocrine outpatient clinics in Switzerland, limiting its generalizability, for instance, to other countries. Most of our patients were treated with liraglutide and dulaglutide (93\%), which may not represent the situation in other regions in the world. This may therefore limit assertions for switches from other GLP-1 analogues to semaglutide, but the treatment effect remained comparable in all subgroup analyses for each previously used GLP-1 analogue. However, liraglutide and dulaglutide are the most frequently used GLP-1 analogues in Switzerland, the European Union and the USA. In addition, eight patients $(10.3 \%)$ received concomitant treatment with an SGLT2 inhibitor (primarily 
because of established cardiovascular disease) which may not be reimbursed in all countries. However, analysing our data without these patients yielded comparable reductions in HbA1c and weight (Supplementary Table 2).

Ethnicity was not recorded in our patient data system, but the majority of patients in our study were Caucasian, which hampers assertions regarding a switch to semaglutide from other GLP-1 analogues in other ethnicities. In addition, the observed treatment period was relatively short for a chronic therapy. However, this period was limited to the time semaglutide was available in Switzerland.

Furthermore, in eight patients, after a switch to semaglutide, the remaining diabetes medication was changed. Comparable to a real-world setting, medication was added in four patients and medication was discontinued during the same period in four patients but did not affect HbA1c and weight reduction at 6 months (Supplementary Table 2).

Prospective studies such as the recently published planned study protocol (SwitchSema-Study) by Nomoto et al. may overcome limitations inherent in our design and will shed further light on this highly clinical question [18].

\section{CONCLUSIONS}

Switching to semaglutide from an established GLP-1 analogue therapy yields a significant reduction in HbA1c and weight loss within 6 months. Switching appears to be reasonable with equipotential dosing to the preceding GLP1 analogue treatment and appears to be well tolerated.

\section{ACKNOWLEDGEMENTS}

First of all, we thank all the patients for participating in this study. We thank Susanne Rüsch, Study Nurse at the University Hospital Basel, and Gabriela Schenker, Study Coordinator at the Cantonal Hospital Olten, for their administrative assistance and Dr Gilles Dulith, PhD, from the Department of Clinical Research at the
University of Basel, for discussion of statistical aspects. Gottfried Rudofsky is the guarantor of the clinical content of this submission.

Funding. No funding or sponsorship was received for this study or publication of this article. The Rapid Service Fee was funded by the authors.

Authorship. All named authors meet the International Committee of Medical Journal Editors (ICMJE) criteria for authorship for this article, take responsibility for the integrity of the work as a whole, and have given their approval for this version to be published.

Author Contributions. GR, DZ and MH planned the study. MH, DZ and MR collected the data. MH performed the statistical analysis and wrote the first draft of the manuscript. All authors participated in the interpretation of the data, critical review of the manuscript, and decision to submit for publication.

Prior Presentation. Parts of this work were presented at the annual meeting of the Swiss Society of Endocrinology and Diabetes, in November 2020, Bern, Switzerland.

Disclosures. Matthias Hepprich, Daniela Zillig, Manuel A. Florian-Reynoso, Marc Y. Donath and Gottfried Rudofsky declare no competing interests.

Compliance with Ethics Guidelines. The study was conducted in compliance with the current version of the Declaration of Helsinki, the ICH-GCP and national legal and regulatory requirements. The study was approved by the local ethics committee (EKNZ 2019-01770).

Data Availability. The dataset will be made publicly available online after publication in the Open Science Framework.

Open Access. This article is licensed under a Creative Commons Attribution-NonCommercial 4.0 International License, which permits any non-commercial use, sharing, adaptation, distribution and reproduction in any medium 
or format, as long as you give appropriate credit to the original author(s) and the source, provide a link to the Creative Commons licence, and indicate if changes were made. The images or other third party material in this article are included in the article's Creative Commons licence, unless indicated otherwise in a credit line to the material. If material is not included in the article's Creative Commons licence and your intended use is not permitted by statutory regulation or exceeds the permitted use, you will need to obtain permission directly from the copyright holder. To view a copy of this licence, visit http://creativecommons.org/licenses/by$\mathrm{nc} / 4.0 /$.

\section{REFERENCES}

1. Almandoz JP, Lingvay I, Morales J, Campos C. Switching between glucagon-like peptide-1 receptor agonists: rationale and practical guidance. Clin Diabetes. 2020;38(4):390-402.

2. Jain AB, Ali A, Gorgojo Martínez JJ, et al. Switching between GLP-1 receptor agonists in clinical practice: expert consensus and practical guidance. Int J Clin Pract. 2020:e13731.

3. Davies MJ, D'Alessio DA, Fradkin J, et al. Management of hyperglycemia in type 2 diabetes, 2018. A consensus report by the American Diabetes Association (ADA) and the European Association for the Study of Diabetes (EASD). Diabetes Care. 2018;41(12):2669-701.

4. Andersen A, Lund A, Knop FK, Vilsbøll T. Glucagonlike peptide 1 in health and disease. Nat Rev Endocrinol. 2018;14(7):390-403.

5. Nauck MA, Quast DR, Wefers J, Meier JJ. GLP-1 receptor agonists in the treatment of type 2 diabetes-state-of-the-art. Mol Metab. 2020:101102. https://doi.org/10.1016/j.molmet.2020.101102

6. Wang Q, Liu L, Gao L, Li Q. Cardiovascular safety of GLP-1 receptor agonists for diabetes patients with high cardiovascular risk: a meta-analysis of cardiovascular outcomes trials. Diabetes Res Clin Pract. 2018;143:34-42.

7. Del Olmo-Garcia MI, Merino-Torres JF. GLP-1 receptor agonists and cardiovascular disease in patients with type 2 diabetes. J Diabetes Res. 2018;2018:4020492.
8. Nauck MA, Petrie JR, Sesti G, et al. A phase 2, randomized, dose-finding study of the novel onceweekly human GLP-1 analog, semaglutide, compared with placebo and open-label liraglutide in patients with type 2 diabetes. Diabetes Care. 2016;39(2):231-41.

9. Pratley RE, Aroda VR, Lingvay I, et al. Semaglutide versus dulaglutide once weekly in patients with type 2 diabetes (SUSTAIN 7): a randomised, openlabel, phase $3 \mathrm{~b}$ trial. Lancet Diabetes Endocrinol. 2018;6(4):275-86.

10. Ahmann AJ, Capehorn M, Charpentier G, et al. Efficacy and safety of once-weekly semaglutide versus exenatide ER in subjects with type 2 diabetes (SUSTAIN 3): a 56-week, open-label randomized clinical trial. Diabetes Care. 2018;41(2):258-66.

11. O'Neil PM, Birkenfeld AL, McGowan B, et al. Efficacy and safety of semaglutide compared with liraglutide and placebo for weight loss in patients with obesity: a randomised, double-blind, placebo and active controlled, dose-ranging, phase 2 trial. Lancet. 2018;392(10148):637-49.

12. Goncalves E, Bell DS. Efficacy of semaglutide versus liraglutide in clinical practice. Diabetes Metab. 2020;46(6):515-17.

13. Overgaard RV, Lindberg S, Thielke D. Impact on HbA1c and body weight of switching from other GLP-1 receptor agonists to semaglutide: a modelbased approach. Diabetes Obes Metab. 2019;21(1): $43-51$.

14. Ikushima I, Jensen L, Flint A, Nishida T, Zacho J, Irie $S$. A randomized trial investigating the pharmacokinetics, pharmacodynamics, and safety of subcutaneous semaglutide once-weekly in healthy male Japanese and Caucasian subjects. Adv Ther. 2018;35(4):531-44.

15. Lingvay I, Desouza CV, Lalic KS, et al. A 26-week randomized controlled trial of semaglutide once daily versus liraglutide and placebo in patients with type 2 diabetes suboptimally controlled on diet and exercise with or without metformin. Diabetes Care. 2018;41(9):1926-37.

16. Marso SP, Holst AG, Vilsbøll T. Semaglutide and cardiovascular outcomes in patients with type 2 diabetes. N Engl J Med. 2017;376(9):891-2.

17. Sorli C, Harashima SI, Tsoukas GM, et al. Efficacy and safety of once-weekly semaglutide monotherapy versus placebo in patients with type 2 diabetes (SUSTAIN 1): a double-blind, randomised, placebocontrolled, parallel-group, multinational, multicentre phase 3 a trial. Lancet Diabetes Endocrinol. 2017;5(4):251-60. 
18. Nomoto H, Oba-Yamamoto C, Takahashi Y, et al. Effects of switching from liraglutide or dulaglutide to subcutaneous semaglutide on glucose metabolism and treatment satisfaction in patients with type 2 diabetes: protocol for a multicenter, prospective, randomized, open-label, blinded-endpoint, parallel-group comparison study (The SWITCH-SEMA 1 Study). Diabetes Ther. https://doi. org/10.1007/s13300-020-00986-9. 